\title{
Logarithmic Aggregation Operators and Distance Measures
}

\author{
Víctor G. Alfaro-García ${ }^{1}$, José M. Merigó ${ }^{2, *}$, Anna M. Gil-Lafuente ${ }^{1}$, Janusz Kacprzyk ${ }^{3}$
}

${ }^{1}$ Department of Business Administration, University of Barcelona, Av. Diagonal 690, 08034 Barcelona, Spain.

${ }^{2}$ Department of Management Control and Information Systems, University of Chile, Av. Diagonal Paraguay 257, 8330015 Santiago, Chile.

${ }^{3}$ Intelligent Systems Laboratory, Polish Academy of Sciences, Newelska 6, 01-447 Warsaw, Poland.

The Hamming distance is a well-known measure that is designed to provide insights into the similarity between two strings of information. In this study, we use the Hamming distance, the optimal deviation model, and the generalized ordered weighted logarithmic averaging (GOWLA) operator to develop the ordered weighted logarithmic averaging distance (OWLAD) operator and the generalized ordered weighted logarithmic averaging distance (GOWLAD) operator. The main advantage of these operators is the possibility of modeling a wider range of complex representations of problems under the assumption of an ideal possibility. We study the main properties, alternative formulations and families of the proposed operators. We analyze multiple classical measures to characterize the weighting vector and propose alternatives to deal with the logarithmic properties of the operators. Furthermore, we present generalizations of the operators, which are obtained by studying their weighting vectors and the lambda parameter. Finally, an illustrative example regarding innovation project management measurement is proposed, in which a multi-expert analysis and several of the newly introduced operators are utilized.

\footnotetext{
* To whom correspondence should be addressed: email: jmerigo@fen.uchile.cl
} 


\section{INTRODUCTION}

Group decision-making (GDM) techniques have increased in relevance in the literature. This is mainly due to the possibility of generating rankings of diverse alternatives for specific situations while considering multiple scenarios. GDM techniques have been widely combined with the theory of aggregation, thereby producing a vast pool of contributions in diverse fields of knowledge such as artificial intelligence, fuzzy systems, imaging processing and decision sciences. This last field is of special interest in our study, as it provides a basis for combining data and obtaining solutions that are constructed based on information that is collected directly from decision makers, experts or stakeholders. There are many aggregation operators and aggregation functions ${ }^{1-4}$ that have proven useful in diverse areas, e.g., statistics, economics, education, biology, computer science and engineering ${ }^{1,2}$. A classic example of an operator that is designed for the aggregation of information in intelligent systems is the ordered weighted average (OWA), which is presented in Yager $^{5}$. The OWA allows for a descending and ascending ordered aggregation mechanism, thereby yielding a result that is between the minimum and the maximum of the values to be combined. It also provides a family of parameterized operators, which have been adopted in several areas such as expert systems, database systems, operational research and fuzzy systems ${ }^{6,7}$.

Recently, the use of distance measurement techniques in the field of group GDM has gained special relevance. The idea of providing results based on the comparison of information that is retrieved from domain experts and an ideal collection of preferences is highly appealing . $^{8}$ The current literature has extensively studied several distance measures, such as the Hamming distance, the Euclidean distance and the Hausdorff distance ${ }^{9-11}$. We focus on the Hamming distance $^{12}$, which considers the importance of each deviation value. This distance has become very popular and is applied in the field of aggregation operators, e.g., in the ordered weighted 
distance (OWD) measures ${ }^{13}$, the ordered weighted averaging distance (OWAD) operators ${ }^{14}$, the linguistic ordered weighted averaging distance (LOWAD) operators ${ }^{15}$, and the induced ordered weighted averaging distance (IOWAD) operators ${ }^{16}$. These studies have motivated research on generating additional applications, such as the intuitionistic fuzzy ordered weighted distance (IFOWD) operator ${ }^{17}$, the fuzzy ordered distance measures that are presented in $^{18}$, a continuous ordered weighted distance (COWD) operator for investment selection problems ${ }^{19}$, a probabilistic ordered weighted averaging distance operator in political management ${ }^{20}$, the linguistic induced ordered weighted averaging distance operators for the selection of investments $^{21}$, distance measures with heavy aggregation operators (HOWAD) for strategic management ${ }^{22}$, a linguistic continuous ordered weighted distance (LCOWD) measure for a GDM in an investment selection problem ${ }^{23}$ and, more recently, the fuzzy linguistic induced ordered weighted averaging Minkowski distance (FLIOWAMD), which generalizes the Euclidean and Hamming distances for investment strategy decision making ${ }^{24}$.

Motivated by the recent work of Zhou and Chen ${ }^{25}$, which proposes an operator that is based on an optimal deviation model and is called the generalized ordered weighted logarithmic aggregation (GOWLA) operator, this study introduces the ordered weighted logarithmic averaging distance (OWLAD) operator and the generalized ordered weighted logarithmic averaging distance (GOWLAD) operator. These operators utilize the Hamming distance measure to provide a set of parameterized families between the maximum and the minimum values, including the step-OWLAD operator, the NLHD operator, the WLHD operator, the olympic-OWLAD, the window-OWLAD operator, the median-OWLAD operator, the centered-OWLAD, the WLGAD operator, the OWLHAD operator, the OWLAD operator, the OWLQAD operator and the OWLCAD operator. These families enable the assessment of complex GDM problems in which a set of optimal preferences must be satisfied while considering diverse alternatives, scenarios and preferences. An increasing number of studies 
are being performed on logarithmic aggregation operators, such as the generalized ordered weighted logarithmic proportional averaging (GOWLPA) operator ${ }^{26}$, the generalized ordered weighted exponential proportional aggregation (GOWEPA) operator ${ }^{27}$, and the generalized ordered weighted logarithmic harmonic averaging (GOWLHA) operator ${ }^{28}$.

The remainder of the paper is organized as follows. In section 2, we present the preliminaries of this study. In section 3, we introduce the OWLAD operators, study their main properties and alternative formulations, propose measures to characterize the weighting vector and introduce families of the operator. Similarly, section 4 presents the study of the GOWLAD operators. Section 5 proposes a decision-making problem in an innovation project management application, which is further assessed with a numerical example in section 6 . Finally, section 7 presents our conclusions.

\section{PRELIMINARIES}

The ordered weighted averaging (OWA) operator ${ }^{5}$ describes a parameterized family of aggregation operators, which include the maximum, the minimum and the average criteria. Applications of this operator have been widely studied in the literature ${ }^{6}$.

The Hamming distance ${ }^{12}$ has become a standard technique to measure the difference between two parameters, elements or sets. This metric has been applied in several domains of knowledge; some of the most well-recognized are fuzzy sets, artificial intelligence, operations research and engineering ${ }^{13}$.

Motivated by the application of aggregation operators to calculate the Hamming distance, Merigó and Gil-Lafuente ${ }^{14}$ and $\mathrm{Xu}$ and $\mathrm{Chen}{ }^{13}$ present the ordered weighted averaging distance (OWAD) operators. The OWAD operators provide a parameterized family of distance aggregation operators between the maximum and the minimum values. 
The generalized ordered weighted logarithmic aggregation (GOWLA) operator was developed by Zhou and Chen ${ }^{25}$. This operator has as its foundation the next optimal model:

$$
\min J_{1}=\sum_{j=1}^{n} w_{j}\left[(\ln y)^{\lambda}-\left(\ln a_{j}\right)^{\lambda}\right]^{2},
$$

where $y$ is an aggregation operator of dimension $n$ and $w=\left(w_{1}, w_{2}, \ldots, w_{n}\right)^{T}$ an associated weighting vector such that $w_{j} \in[0,1]$ for all $j$ and $\sum_{j=1}^{n} w_{j}=1$. Observe that $\lambda \in(-\infty, \infty)$. By calculating the partial derivative respect to $y$ and $\frac{\partial y_{1}}{\partial y}=0$, we obtain the generalized weighted logarithmic averaging (GWLA) operator:

$$
G W L A\left(a_{1}, a_{2}, \ldots, a_{n}\right)=\exp \left\{\left(\sum_{j=1}^{n} w_{j}\left(\ln a_{j}\right)^{\lambda}\right)^{\frac{1}{\lambda}}\right\}
$$

By reordering the arguments $a_{i}$, we obtain the generalized ordered weighted logarithmic averaging (GOWLA) operator, as follows:

DEFINITION 1. A GOWLA operator of dimension $n$ is a mapping GOWLA: $\Omega^{n} \rightarrow \Omega$ with an associated weighting vector $w$ of dimension $n$, such that $w_{j} \in[0,1]$ for all $j$ and $\sum_{j=1}^{n} w_{j}=1$, which includes a parameter $\lambda$ in the range of $(-\infty, \infty)-\{0\}$ and satisfies the following formula:

$$
\operatorname{GOWLA}\left(a_{1}, a_{2}, \ldots, a_{n}\right)=\exp \left\{\left(\sum_{j=1}^{n} w_{j}\left(\ln b_{j}\right)^{\lambda}\right)^{\frac{1}{\lambda}}\right\}
$$

where $b_{j}$ is the $j$ th largest of the arguments $a_{1}, a_{2}, \ldots, a_{n}$. From $\ln a_{j} \geq 0$, it follows that $\exp \left(\ln a_{j}\right) \geq \exp (0)$. Thus, $a_{j} \geq 1$. In the present paper, we follow the original notation ${ }^{25}$ : $\Omega=\{x \mid x \geq 1, x \in R\}$. 
An interesting family of the GWLA operator results when parameter $\lambda=1$. In this case, we obtain an extension, which is called the weighted logarithmic aggregation (WLA) operator. We define the WLA operator as follows:

$$
W L A\left(a_{1}, a_{2}, \ldots, a_{n}\right)=\exp \sum_{j=1}^{n} w_{i}\left(\ln a_{i}\right)
$$

\section{ORDERED WEIGHTED LOGARITHMIC AVERAGING DISTANCE OPERATORS}

\subsection{Weighted logarithmic averaging distance operator}

The WLAD operator is a distance measure that is based on the optimal deviation model, which was proposed by Zhou and Chen ${ }^{25}$. It uses the Hamming distance to obtain a result that is between the minimum and maximum values that are considered in the problem.

DEFINITION 2. A WLAD operator of dimension $n$ is a mapping WLAD: $\Omega^{n} \times \Omega^{n} \rightarrow \Omega$ that is defined by an associated weighting vector $W$ such that the sum of the weights is equal to 1 and $w_{j} \in[0,1]$, according to the following formula:

$$
W L A D\left(\left\langle x_{1}, y_{1}\right\rangle,\left\langle x_{2}, y_{2}\right\rangle, \ldots,\left\langle x_{n}, y_{n}\right\rangle\right)=\exp \left\{\sum_{j=1}^{n} w_{j}\left(\ln \left|x_{i}-y_{i}\right|\right)\right\}
$$

where the argument $\left|x_{i}-y_{i}\right|$ is a variable that is represented in the form of an individual distance.

In this paper, we follow the original definition ${ }^{25}$ of $\Omega=\{x \mid x \geq 1, x \in R\}$. If the individual distance $\left|x_{i}-y_{i}\right|=0$, it is not possible to carry out the aggregation process because 
in logarithmic aggregation, we cannot use values that are less than 1 . Therefore, we do not consider individual distances that are less than 1 in the aggregation, i.e., they are considered empty.

EXAMPLE 1. Assume the following collection of arguments: $X=(9,24,11,33), Y=$ $(12,15,28,23)$, and $W=(0.4,0.1,0.3,0.2)$. The aggregation has the following result:

$$
\begin{aligned}
W L A D(X, Y)= & \exp \{0.4 \times(\ln |9-12|)+0.1 \times(\ln |24-15|)+0.3 \times(\ln |11-28|)+0.2 \\
& \times(\ln |33-23|)\}=7.1682
\end{aligned}
$$

An alternative formulation to this approach is:

$$
W L A D\left(\left\langle x_{1}, y_{1}\right\rangle,\left\langle x_{2}, y_{2}\right\rangle, \ldots,\left\langle x_{n}, y_{n}\right\rangle\right)=\exp \left\{\sum_{j=1}^{n} w_{j}\left|\ln \left(x_{i}\right)-\ln \left(y_{i}\right)\right|\right\}
$$

\subsection{Ordered weighted logarithmic averaging distance operator}

The OWLAD operator is a generalization of the WLAD operator. The most distinctive property is the ordering mechanism of the considered arguments. This order enables the introduction of complex decision-making processes. Additionally, it generates the possibility of having alternative formulations that depend not only on the ascending or descending direction of the ordering mechanism but also on the system that is designed to solve the logarithmic distances. The main properties of the OWLAD operator are commutativity, idempotency, boundedness, monotonicity and non-negativity. 
DEFINITION 3. An ordered weighted logarithmic averaging distance (OWLAD) operator of dimension $n$ is a mapping OWLAD: $\Omega^{n} \times \Omega^{n} \rightarrow \Omega$ that has an associated weighting vector $W$, with $\sum_{j=1}^{n} w_{j}=1$ and $w_{j} \in[0,1]$, such that:

$$
O W L A D\left(\left\langle x_{1}, y_{1}\right\rangle,\left\langle x_{2}, y_{2}\right\rangle, \ldots,\left\langle x_{n}, y_{n}\right\rangle\right)=\exp \left\{\sum_{j=1}^{n} w_{j} \ln \left(D_{j}\right)\right\},
$$

where $D_{j}$ represents the $j$ th largest of $\left|x_{i}-y_{i}\right|$ over all i and $\left|x_{i}-y_{i}\right|$ is the argument variable, which is represented in the form of individual distances.

EXAMPLE 2. Assume the same collection of arguments as was defined in Example 1: $X=$ $(9,24,11,33), Y=(12,15,28,23)$, and $W=(0.4,0.1,0.3,0.2)$. Then, the aggregation will yield the following result:

$O W L A D(X, Y)=\exp \{0.4 \times(\ln |11-28|)+0.1 \times(\ln |33-23|)+0.3 \times(\ln |24-15|)+$ $0.2 \times(\ln |9-12|)\}=9.4162$.

From the ordering mechanism perspective, which differentiates this operator from the WLAD operator, two formulations can be described: the descending ordered weighted logarithmic averaging distance (DOWLAD) operator and the ascending ordered weighted logarithmic averaging distance (AOWLAD) operator. The relation between these operators is $w_{j}=w^{*}{ }_{n+1-j}$, where $w_{j}$ is the $j$ th weight of the DOWLAD operator and $w^{*}{ }_{n+1-j}$ is the $j$ th weight of the AOWLAD operator.

In the presence of non-normalization in the arguments, i.e., $W=\sum_{j=1}^{n} w_{j} \neq 1$ (see ${ }^{1}$ ), the OWLAD operator can be expressed as: 


$$
O W L A D\left(\left\langle x_{1}, y_{1}\right\rangle,\left\langle x_{2}, y_{2}\right\rangle, \ldots,\left\langle x_{n}, y_{n}\right\rangle\right)=\exp \left\{\frac{1}{W} \sum_{j=1}^{n} w_{j} \ln \left(D_{j}\right)\right\}
$$

where $W=\sum_{j}^{n} w_{j}$.

The ordered weighted logarithmic aggregation operator has the following main properties: commutativity, idempotency, boundedness, monotonicity and non-negativity. The proofs of these properties are trivial. Therefore, they are omitted. These properties can be expressed by the following theorems:

THEOREM 1. Commutativity, by the ordered weighted aggregation. Let the function $f$ be the OWLAD operator. Then,

$$
f\left(\left\langle x_{1}, y_{1}\right\rangle, \ldots,\left\langle x_{n}, y_{n}\right\rangle\right)=f\left(\left\langle c_{1}, d_{1}\right\rangle, \ldots,\left\langle c_{n}, d_{n}\right\rangle\right),
$$

where $\left(\left\langle x_{1}, y_{1}\right\rangle, \ldots,\left\langle x_{n}, y_{n}\right\rangle\right)$ represents any specified permutation of the arguments $\left(\left\langle c_{1}, d_{1}\right\rangle, \ldots,\left\langle c_{n}, d_{n}\right\rangle\right)$

THEOREM 2. Commutativity, by the distance measure. Assume $f$ is the OWLAD operator. Then,

$$
f\left(\left\langle x_{1}, y_{1}\right\rangle, \ldots,\left\langle x_{n}, y_{n}\right\rangle\right)=f\left(\left\langle x_{1}, y_{1}\right\rangle, \ldots,\left\langle x_{n}, y_{n}\right\rangle\right)
$$

THEOREM 3. Monotonicity. Let $f$ be the OWLAD operator. If $\left|x_{i}-y_{i}\right| \geq\left|c_{i}-d_{i}\right|$ for all $i$, then

$$
f\left(\left\langle x_{1}, y_{1}\right\rangle, \ldots,\left\langle x_{n}, y_{n}\right\rangle\right) \geq f\left(\left\langle c_{1}, d_{1}\right\rangle, \ldots,\left\langle c_{n}, d_{n}\right\rangle\right)
$$

THEOREM 4. Boundedness. Assume the function $f$ is the OWLAD operator. Then,

$$
\min \left\{\left|x_{i}-y_{i}\right|\right\} \leq f\left(\left\langle x_{1}, y_{1}\right\rangle, \ldots,\left\langle x_{n}, y_{n}\right\rangle\right) \leq \max \left\{\left|x_{i}-y_{i}\right|\right\}
$$

THEOREM 5. Idempotency. If the function $f$ is the OWLAD operator and $\left|x_{i}-y_{i}\right|=a_{i}$ for all $i$, then

$$
f\left(\left\langle x_{1}, y_{1}\right\rangle, \ldots,\left\langle x_{n}, y_{n}\right\rangle\right)=a
$$

THEOREM 6. Non-negativity. Let the function $f$ to be the OWLAD operator. Then, 


$$
f\left(\left\langle x_{1}, y_{1}\right\rangle, \ldots,\left\langle x_{n}, y_{n}\right\rangle\right) \geq 0
$$

\subsection{Alternative formulations of the OWLAD operators}

Depending on the ordering of the arguments in the aggregation process, four alternative formulations can be generated for the OWLAD operator:

1) The $O W L A D^{I}$ operator can be obtained by solving $\left|x_{i}-y_{i}\right|$, calculating the natural logarithm of the difference, and ordering the arguments in a descending direction, according to the following formula:

$$
O W L A D^{I}\left(\left\langle x_{1}, y_{1}\right\rangle,\left\langle x_{2}, y_{2}\right\rangle, \ldots,\left\langle x_{n}, y_{n}\right\rangle\right)=\exp \left\{\sum_{j=1}^{n} w_{j} \ln \left(D_{j}\right)\right\}
$$

where $D_{j}$ represents the $j$ th largest of $\left|x_{i}-y_{i}\right|$ over all i and $\left|x_{i}-y_{i}\right|$ is the argument variable, which is represented in the form of individual distances. Note that this alternative formulation is equivalent to Eq. (10).

2) The $O W L A D^{I I}$ operator is generated by finding the natural logarithm of each argument, i.e., $\ln \left(x_{i}\right)$ and $\ln \left(y_{i}\right)$; finding the absolute difference of the obtained results; and ordering the arguments in a descending direction, according to the following formula:

$$
O W L A D^{I I}\left(\left\langle x_{1}, y_{1}\right\rangle,\left\langle x_{2}, y_{2}\right\rangle, \ldots,\left\langle x_{n}, y_{n}\right\rangle\right)=\exp \left\{\sum_{j=1}^{n} w_{j}\left|S_{j}-B_{j}\right|\right\}
$$

where $S_{j}$ represents the $j$ th largest of $\ln \left(x_{i}\right)$ over all $i$ and $B_{j}$ represents the $j$ th largest of $\ln \left(y_{i}\right)$ over all $i$. Both arguments are ordered in a descending way. 
3) The $O W L A D^{I I I}$ operator is obtained when we order arguments $x_{i}$ and $y_{i}$ in a descending way, calculate the absolute difference of the ordered arguments, and calculate the natural logarithm of the results. This sequence of steps can be formulated as:

$$
O W L A D^{I I I}\left(\left\langle x_{1}, y_{1}\right\rangle,\left\langle x_{2}, y_{2}\right\rangle, \ldots,\left\langle x_{n}, y_{n}\right\rangle\right)=\exp \left\{\sum_{j=1}^{n} w_{j} \ln \left(\left|E_{j}-M_{j}\right|\right)\right\}
$$

where $E_{j}$ represents the $j$ th largest of $x_{i}$ over all $i$ and $M_{j}$ represents the $j$ th largest of $y_{i}$ over all $i$. Both arguments are ordered in a descending way.

4) The $O W L A D^{I V}$ operator is obtained when we order arguments $x_{i}$ and $y_{i}$ in a descending way, calculate the natural logarithm of the ordered arguments, and find the distance of the results. This mechanism can be formulated as:

$$
\begin{aligned}
O W L A D^{I V}\left(\left\langle x_{1}, y_{1}\right\rangle,\left\langle x_{2}, y_{2}\right\rangle, \ldots,\left\langle x_{n}, y_{n}\right\rangle\right) \\
\quad=\exp \left\{\sum_{j=1}^{n} w_{j}\left\{\left[\ln \left(E_{j}\right)\right]-\left[\ln \left(M_{j}\right)\right]\right\}\right\},
\end{aligned}
$$

where $E_{j}$ represents the $j$ th largest of $x_{i}$ over all $i$ and $M_{j}$ represents the $j$ th largest of $y_{i}$ over all $i$. Both arguments are ordered in a descending way.

EXAMPLE 3. Following the same arguments as in Example 1, the results for each alternative formulation of the OWLAD operator are described in Table I.

Insert Table I about here

\subsection{Characterization of OWLAD operators}


Multiple approaches have been proposed in the literature to measure and thus characterize the weights of aggregation functions. The classical methods include, e.g., the degree of orness ${ }^{5}$, the dispersion measure ${ }^{5,29}$, the balance and the divergence. In the case of the OWLAD operator, additional measures must be developed, as the logarithmic properties of the aggregation limit the consideration of numbers between 0 and 1 . Motivated by this, we propose a general characterization of the weighting vector and a transformation of the OWA measures into the R-scale.

\subsubsection{General characterization of the aggregation}

Since logarithms do not work in the scale $[0,1]$, we must find additional measures to characterize the aggregation. A general approach to characterize the descending aggregation (CDA) is formulated as follows:

$$
C D A=\frac{b_{j}-b_{n}}{b_{1}-b_{n}}
$$

where $b_{j}$ is the result of the OWLAD operator and $b_{1}$ and $b_{n}$ are the largest and smallest arguments of $\left|x_{i}-y_{i}\right|$, respectively. This approach requires the aggregation results to be ordered in a descending way. Furthermore, the dual version of this formulation can be represented as:

$$
C D A+C D A^{*}=1
$$

Then,

$$
C D A^{*}=1-\frac{b_{j}-b_{n}}{b_{1}-b_{n}}
$$

If the aggregation results are ordered in an ascending way, the formula for the characterization of the ascending aggregation (CAA) needs to be changed to the following: 


$$
C A A=\frac{b_{j}-b_{1}}{b_{n}-b_{1}}
$$

where $b_{j}$ is the result of the OWLAD operator and $b_{1}$ and $b_{n}$ are the largest and smallest arguments of $\left|x_{i}-y_{i}\right|$, respectively. As presented for the descending formulation, the dual version of this representation can be obtained as:

$$
C A A^{*}=1-\frac{b_{j}-b_{1}}{b_{n}-b_{1}}
$$

EXAMPLE 4. We utilize the values that were defined in Example 2: $X=(9,24,11,33), Y=$ $(12,15,28,23)$, and $W=(0.4,0.1,0.3,0.2)$. The general characterization results of the aggregation and their dual versions are presented in Table II.

Insert Table II about here

\subsubsection{Transformation of the OWA measures into the R-scale}

An interesting mechanism for characterizing the weighting vector, including the logarithmic properties of the weighted logarithmic aggregation operators, is the transformation of the OWA measures into the R-scale. The proposed procedure can be realized by the following steps.

Let $\mathrm{Z}$ be the transformation of the aggregation arguments according to the following expression:

$$
Z=\min +\{\max -\min \}\left(\frac{n-j}{n-1}\right)
$$


Observe that the use of $Z$ enables the transformation of the $[0,1]$ scale into a logarithmically consistent one. Motivated by the result of this procedure, we propose using the $Z$ transformation to study the degree of orness of the OWLA operator.

STEP 1. Calculate $R-\alpha(w)$, which includes the $Z$ transformation, using the following equation:

$$
R-\alpha(w)=e\left\{\sum_{j=1}^{n} w_{j} \ln \left(Z_{j}\right)\right\}
$$

where $Z$ is the transformation of the arguments in the aggregation. The complete formulation can be expressed as:

$$
R-\alpha(w)=e\left\{\sum_{j=1}^{n} w_{j} \ln \left(\min \left(a_{i}\right)+\left\{\max \left(a_{i}\right)-\min \left(a_{i}\right)\right\}\left(\frac{n-j}{n-1}\right)\right)\right\},
$$

where $a_{i}$ is the argument $\left|x_{i}-y_{i}\right|$ of the aggregation.

STEP 2. The final step is to convert the result $R-\alpha(w)$ using the following expression:

$$
x=\frac{y-\min \left(a_{i}\right)}{\left(\max \left(a_{i}\right)-\min \left(a_{i}\right)\right)}
$$

where $y$ is the result of $R-\alpha(w)$ and $x \in[0,1]$. The minimum is attained when $x=0$, and the maximum, when $x=1$. We can obtain the dual of this operation by applying the following formulation.

Let $x^{*}$ be the dual of $x$. Then, 


$$
x+x^{*}=1
$$

It follows that

$$
x^{*}=1-\frac{y-\min \left(a_{i}\right)}{\left(\max \left(a_{i}\right)-\min \left(a_{i}\right)\right)}=\frac{\max \left(a_{i}\right)-y}{\max \left(a_{i}\right)-\min \left(a_{i}\right)} .
$$

EXAMPLE 5. We utilize the arguments that are defined in Example $2: X=(9,24,11,33)$, $Y=(12,15,28,23)$, and $W=(0.4,0.1,0.3,0.2)$. Then, the degree of orness in the logarithmic scale is as follows:

$$
\begin{aligned}
R-\alpha(w)= & e\{0.4[\ln (3+14(1))]+0.1[\ln (3+14(0.6667))]+0.3[\ln (3+14(0.3333))] \\
& +0.2[\ln (3+14(0))]\}=9.1642 .
\end{aligned}
$$

Therefore,

$$
x=\frac{9.1642-3}{(17-3)}=0.4403
$$

and

$$
x^{*}=\frac{17-9.1642}{17-3}=0.5597
$$

It is interesting to study the families of the OWLAD operators, as they represent particular cases that can be selected in accordance with specific problems that we are assessing. For the case of the OWLAD operator, several parameterized families can be described, depending on the conformation of the weighting vector ${ }^{30}$. These particular families include the maximum and minimum distances, the step-OWLAD operator, the normalized logarithmic Hamming distance (NLHD), the weighted logarithmic Hamming distance (WLHD), the 
olympic-OWLAD, the window-OWLAD operator, the median-OWLAD operator, and the centered-OWLAD ${ }^{31}$ operator. Note that all the alternative formulations that were described previously are also applicable to the families that are presented here ${ }^{32-34}$.

\section{GENERALIZED ORDERED WEIGHTED LOGARITHMIC DISTANCE OPERATORS}

\subsection{Generalized weighted logarithmic averaging distance operator}

The GWLAD operator is a generalization of the OWLAD operator. Therefore, it shares the same properties and characteristics. The GWLAD operator includes a $\lambda$ parameter, which allows for a wider representation of complex problems. Many interesting families of the GWLAD can be developed, depending on the $\lambda$ value.

DEFINITION 4. A GWLAD operator of dimension $n$ is a mapping GWLAD: $\Omega^{n} \times \Omega^{n} \rightarrow \Omega$ with an associated weighting vector $W$ of dimension $n$ such that the sum of all $w_{j}$ is equal to 1 , and $w_{j} \in[0,1]$. It is expressed by the following formula:

$$
\begin{aligned}
G W L A D\left(\left\langle x_{1}, y_{1}\right\rangle,\left\langle x_{2}, y_{2}\right\rangle, \ldots,\left\langle x_{n}, y_{n}\right\rangle\right) \\
=\exp \left\{\left(\sum_{j=1}^{n} w_{j}\left(\ln \left|x_{i}-y_{i}\right|\right)^{\lambda}\right)^{\frac{1}{\lambda}}\right\},
\end{aligned}
$$

where $\left|x_{i}-y_{i}\right|$ is an argument variable, which is represented in the form of an individual distance, and $\lambda$ is a parameter such that $\lambda \in(-\infty, \infty)-\{0\}$. 
If $w_{j}=\frac{1}{n}$ for all $j$, we obtain the generalized logarithmic averaging distance operator (GLAD), which is formulated as follows:

$$
G L A D\left(\left\langle x_{1}, y_{1}\right\rangle,\left\langle x_{2}, y_{2}\right\rangle, \ldots,\left\langle x_{n}, y_{n}\right\rangle\right)=\exp \left\{\left(\frac{1}{n} \sum_{j=1}^{n}\left(\ln \left|x_{i}-y_{i}\right|\right)^{\lambda}\right)^{\frac{1}{\lambda}}\right\}
$$

EXAMPLE 6. We utilize the arguments that were defined in Example 2, namely, $X=$ $(9,24,11,33), Y=(12,15,28,23)$, and $W=(0.4,0.1,0.3,0.2)$, as well as parameter $\lambda=2$. The aggregation yields:

$$
\begin{aligned}
G W L A D(X, Y) & =\exp \left\{\left[0.4 \times(\ln |9-12|)^{2}+0.1 \times(\ln |24-15|)^{2}+0.3 \times(\ln |11-28|)^{2}\right.\right. \\
& \left.\left.+0.2 \times(\ln |33-23|)^{2}\right]^{1 / 2}\right\}=8.2130
\end{aligned}
$$

Additionally, parameter $\lambda$ in the GWLAD operator enables the study of particular cases. Table III presents special cases that are interesting for analysis.

$$
\text { Insert Table III about here }
$$

EXAMPLE 7. We utilize the arguments that were defined in Example 2. The results for each family of the GWLAD operator are shown in Table IV.

Insert Table IIV about here 


\subsection{Generalized ordered weighted logarithmic averaging distance operator}

The GOWLAD operator adds an ordering mechanism to the GWLAD operator. Therefore, as a generalization of the GWLAD operator, it shares the same properties. The ordering mechanism allows for the modeling of a wider range of more complex problems. Additionally, it introduces the possibility of additional alternative formulations and families, depending on the value of $\lambda$.

DEFINITION 5. A GOWLAD operator of dimension $n$ is a mapping GOWLAD: $\Omega^{n} \times \Omega^{n} \rightarrow$ $\Omega$ that is defined by an associated weighting vector $W$ of dimension $n$ such that the sum of the weights is equal to 1 and $w_{j} \in[0,1]$, according to the following formula:

$$
\operatorname{GOWLAD}\left(\left\langle x_{1}, y_{1}\right\rangle,\left\langle x_{2}, y_{2}\right\rangle, \ldots,\left\langle x_{n}, y_{n}\right\rangle\right)=\exp \left\{\left(\sum_{j=1}^{n} w_{j}\left(\ln b_{j}\right)^{\lambda}\right)^{\frac{1}{\lambda}}\right\}
$$

where $b_{j}$ is the $\left|x_{i}-y_{i}\right|$ value of GOWLAD $\left\langle x_{i}, y_{i}\right\rangle$, in decreasing order of the value of $\left|x_{i}-y_{i}\right|$. The argument $\left|x_{i}-y_{i}\right|$ is a variable that is represented in the form of an individual distance, and $\lambda$ is a parameter that satisfies $\lambda \in(-\infty, \infty)-\{0\}$.

EXAMPLE 8. We utilize the arguments that were defined in Example 2: $X=(9,24,11,33)$ and $Y=(12,15,28,23)$. Assuming $W=(0.4,0.1,0.3,0.2)$ and $\lambda=2$, the aggregation yields:

$$
\begin{aligned}
& \operatorname{GOWLAD}(X, Y)=\exp \left\{\left((0.4 \times(\ln |11-28|))^{2}+(0.1 \times(\ln |33-23|))^{2}+(0.3 \times\right.\right. \\
& \left.\left.(\ln |24-15|))^{2}+(0.2 \times(\ln |9-12|))^{2}\right)^{\frac{1}{2}}\right\}=10.2820 .
\end{aligned}
$$


The descending order of arguments $b_{j}$ depends on the result of $\left|x_{i}-y_{i}\right|$.

In addition, the GOWLAD operator is a generalization of the OWLAD operator. Thus, it also has the properties of commutativity, monotonicity, boundedness and idempotency.

Similarly to the OWLAD operator, the GOWLAD operator exhibits four alternative formulations that depend on the ordering of the arguments. Note that obtaining these formulations is straightforward based on section 3.4.

EXAMPLE 9. Following the data that were presented in Examples 7 and 8, the results for each alternative formulation of the GOWLAD operator are described in Table V.

Insert Table V about here

Several particular families of the GOWLAD operator can be delimitated by the values of the parameter $\lambda$. Table VI presents some representative cases of the GOWLAD operator families, including the ordered weighted logarithmic geometric averaging distance (OWLGAD) operator, the ordered weighted logarithmic harmonic averaging distance (OWLHAD) operator, the ordered weighted logarithmic aggregation distance (OWLAD) operator, the ordered weighted logarithmic quadratic aggregation distance (OWLQAD) operator, the ordered weighted logarithmic cubic aggregation distance (OWLCAD) operator, the maximum and the minimum.

Insert Table VI about here 


\section{GROUP DECISION-MAKING IN INNOVATION PROJECT MANAGEMENT}

The GOWLAD operator, which is based on the Hamming distance mechanism, is applicable to a wide range of problems in decision-making procedures. This operator can also

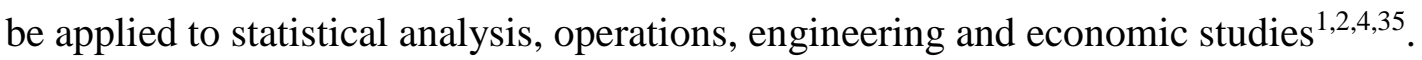

This paper presents a decision-making ${ }^{36-37}$ application in the field of innovation project management ${ }^{38}$. The main motivation for using the GOWLAD operator in this area is the possibility of retrieving the opinions of several experts to select the most efficient solution for a company when managing new projects. Commonly, project management performance has been measured in terms of cost, duration and return over investment ${ }^{38,39}$. However, the GOWLAD operator opens the option to evaluate uncertain and subjective factors such as the extent of internal communication of the implicated areas when developing a new product ${ }^{40}$ and the collaborations with suppliers ${ }^{41}$ and customers ${ }^{42}$, as they have been identified as sources that contribute to the innovation process. The general process to assess a multi-person decisionmaking situation using the GOWLAD operator can be described as follows:

STEP 1. Let $A=\left\{A_{1}, A_{2}, \ldots, A_{m}\right\}$ be a set of limited options, and $C=\left\{C_{1}, C_{2}, \ldots, C_{m}\right\}$, a set of finite options or alternatives. Both sets form a matrix $\left(x_{h i}\right)_{m \times n}$. Let $E=\left\{E_{1}, E_{2}, \ldots, E_{q}\right\}$ be a finite set of decision makers. Assume that the decision makers have diverse levels of importance, where $V=\left(v_{1}, v_{2}, \ldots, v_{p}\right)$ represents the weighting vector of importance, which satisfies $\sum_{k=1}^{p} v_{k}=1$ and $x_{k} \in[0,1]$. At that point, each decision maker must deliver a pay-off $\operatorname{matrix}\left(x_{h i}\right)_{m \times n}^{k}$

STEP 2. Ideal characteristics must be set for the ideal project to be developed; see Table VII. In this case, $P$ is the ideal project, which is represented by a subset; $C_{i}$ represents the $i$ th 
considered characteristic; $y_{i} \in[1,100]$; and $i=1,2, \ldots, n$ is a number between 1 and 100 . Each decision maker must provide an ideal project $y_{i}{ }^{k}$.

\section{Insert Table VII about here}

STEP 3. Apply the weighted average (WA) to aggregate the information of the decision makers $E$ by using the weighting vector $V$. The result will be the collective payoff matrix $\left(x_{h i}-y_{h i}\right)_{m \times n}$. Therefore, $x_{h i}-y_{h i}=\sum_{k=1}^{p} v_{k}\left(x_{h i}^{k}-y_{h i}^{k}\right)$. Note that more complex aggregations can be developed if the experts' opinions are aggregated with a different method than WA, e.g., the OWA operator.

STEP 4. Solve for the GOWLAD operator, as described in Eq. 43. The value of $\lambda$ is usually set to 1 ; however, any of the families that are described in section 4.4 can be used, depending on the problem that is being assessed.

STEP 5. Establish a ranking of the evaluated options, compare the results for the problem that is being assessed and develop a decision-making approach.

To summarize this aggregation mechanism, we propose the utilization of the following aggregation operator, which is named the multi-person-GOWLAD (MP-GOWLAD) operator:

DEFINITION 6. An MP-GOWLAD operator is an aggregation operator with an associated weighting vector $V$ of dimension $p$ such that the sum of the weights is 1 and $v_{k} \in[0,1]$, and a weighting vector $W$ of $n$ dimension such that $\sum_{j=1}^{n} w_{j}=1$ and $w_{j} \in[0,1]$ : 


$$
\begin{gathered}
M P-\operatorname{GOWLAD}\left(\left(x_{1}^{1}, \ldots, x_{1}^{p}\right),\left(y_{1}^{1}, \ldots, y_{1}^{p}\right), \ldots,\left(x_{n}^{1}, \ldots, x_{n}^{p}\right),\left(y_{n}^{1}, \ldots, y_{n}^{p}\right)\right) \\
=\exp \left\{\left(\sum_{j=1}^{n} w_{j}\left(\ln b_{j}\right)^{\lambda}\right)^{\frac{1}{\lambda}}\right\},
\end{gathered}
$$

where $b_{j}$ is the $\left|x_{i}-y_{i}\right|$ value of the MP-GOWLAD $\left(x_{i}, y_{i}\right)$ in decreasing order of the values of the argument $\left|x_{i}-y_{i}\right|$. The argument $\left|x_{i}-y_{i}\right|=\left(\sum_{k=1}^{p} v_{k}\left|x_{i}^{k}-y_{i}^{k}\right|\right)$, where $\left|x_{i}^{k}-y_{i}^{k}\right|$ are variables that correspond to the opinions of each expert in the form of individual distances and $\lambda$ is a parameter that satisfies $\lambda \in(-\infty, \infty)-\{0\}$. The MP-GOWLAD operator shares the properties of the GOWLAD operator.

The MP-GOWLAD operator can be reduced to a series of particular cases by following the methodology that is presented in section 3. Interesting cases include the multi-personnormalized logarithmic Hamming distance (MP-NLHD) operator, the multi-person-weighted logarithmic Hamming distance (MP-WLHD) operator, the multi-person-OWLAD (MPOWLAD) operator, the multi-person-OWLA (MP-OWLA) operator, and the multi-person WLA (MP-WLA) operator.

\section{NUMERICAL EXAMPLE}

This section presents an illustrative example of a strategic decision-making procedure in innovation project management that uses a multi-person analysis and the GOWLAD operator. Observe that additional business-decision-making applications can be assessed, especially in the area of innovation management, which has been widely described as an uncertain and subjective topic. Thus, it is an interesting area for expert decision-making procedures. 
STEP 1. Assume that a real-estate construction company must select the most adequate project to develop from their portfolio of six potential projects:

- $A_{1}$ Industrial park

- $A_{2}$ Small multi-family housing

- $\quad A_{3}$ Residential building

- $A_{4}$ City villas

- $A_{5}$ Commercial building

- $\quad A_{6}$ Luxury apartments

To select the project to be developed, the company chooses diverse experts to evaluate 6 key characteristics:

- $S_{1}$ Cost of the project

- $S_{2}$ Duration

- $S_{3}$ Return on investment (ROI)

- $S_{4}$ Expertise

- $S_{5}$ Internal communication

- $S_{6}$ External communication

A total of three experts are asked for their opinions. The results for each of the projects are shown in Tables VIII-X. All valuations are expressed in terms of numbers between 1 and 100 , where 100 is the maximum valuation.

STEP 2. Representing the objectives of the decision makers, each of the experts constructs the ideal project to be developed. The results of this process are shown in Table XI. 
STEP 3. The weighting vector that represents the importance of each expert in the analysis is $V=(0.5,0.25,0.25)$. With this information, we use the weighted average to aggregate the information into a collective matrix. The results are shown in Table XII.

STEP 4. We apply some of the GOWLAD operator families, aggregate the collective information and obtain the final results. Tables XIII and XIV show the results of the aggregations.

STEP 5. To generate a complete picture of the aggregations, we must establish a ranking of the performance of each project that is based on the preferences of the decision makers. The ordering of alternatives is presented in Table XV. The symbol "\}" denotes "preferred to". Moreover, for each of the selected aggregation operators, a different ranking can be generated. Therefore, distinct decision-making processes will result from that operation.

Insert Table VIIVIII-XV about here

The ranking changes depending on the aggregation mechanism of the chosen operator. In our example, based on the opinions of three experts, the closest options to an ideal project are $A_{5}$ (Commercial building) and $A_{3}$ (Residential building). It is inferred that the company has more experience in developing these real-estate constructions. Moreover, it is implied that the innovative characteristics of the company align in an adequate way with the preferences of the firm. 


\section{CONCLUSIONS}

This paper introduces a new family of ordered weighted logarithmic averaging distance operators, including the ordered weighted logarithmic averaging distance (OWLAD) operator and the generalized ordered weighted logarithmic averaging distance (GOWLAD) operator. The foundation of this approach is the optimal deviation model, which is based on the GOWLA operator. Therefore, it shares the same properties. The main motivation is the extension of its characteristics to consider a wider range of complex problems. The main advantage of the ordered weighted logarithmic averaging distance operators is the introduction of distance measures, specifically the Hamming distance, to consider an optimal set of preferences and compare them to the options or alternatives that are selected by the decision makers.

The OWLAD and GOWLAD operators have diverse properties such as commutativity, idempotency, boundedness, monotonicity, non-negativity and reflexivity. We have studied different classical measures to characterize the weighting vector including the degree of orness, dispersion, balance and divergence measures. Moreover, motivated by the observation that these measures fail to work with numbers that are between 0 and 1 , we propose additional measures to characterize the aggregation, including a transformation of the OWA measures into the R-scale. We have also presented four alternative formulations of the OWLAD and GOWLAD operators, which can be utilized depending on the ordering of the arguments to be aggregated.

Several particular cases of the ordered weighted logarithmic averaging distance operators have been analyzed. First, depending on the conformation of the weighting vector, the OWLAD operator can be reduced to the maximum and minimum distances, the stepOWLAD operator, the NLHD operator, the WLHD operator, the olympic-OWLAD, the window-OWLAD operator, the median-OWLAD operator, and the centered-OWLAD. Second, 
by analyzing the parameter $\lambda$, the GOWLAD operator is found to correspond to specific families, including the maximum and the minimum, the OWLGAD operator, the OWLHAD operator, the OWLAD operator, the OWLQAD operator and the OWLCAD operator.

The OWLAD and GOWLAD operators, including their particular cases and families, are designed to aid group decision-making processes. Engineering, statistics and economics are some of the scientific areas to which this new approach could be applied. To exemplify the use of the OWLAD and GOWLAD operators, we present a multi-person group decision-making problem in the area of innovation project management. The main advantage of this method is the utilization of several experts to assess a complex decision-making procedure that involves objective and subjective factors. Innovation management has been described as an uncertain series of steps and procedures; this makes the topic interesting and viable to analyze. The results in the illustrative example represent different combinations of options and alternatives that depend on the complex attitudinal characteristics of the decision makers among an ideal series of characteristics and enable the comparison among the possible projects to realize.

Additional research is needed to address the main limitations of this study, which are the multifaceted properties of the logarithms, which complicate the development of characterization measures for the weighting vector. In addition, complex decision-making processes such as innovation management require the development of new and robust techniques that consider uncertain information such as fuzzy numbers ${ }^{43}$, linguistic variables ${ }^{37}$, and interval numbers, as well as heavy aggregations of other complex formulations.

\section{Acknowledgements}


The first author expresses his gratitude to the Mexican Council of Science and Technology (CONACYT) for the financial support that was given to this research project with scholarship no. 381436 for graduate studies. The research was supported by "Red Iberoamericana para la Competitividad, Innovación y Desarrollo” (REDCID) project number 616RT0515 in "Programa Iberoamericano de Ciencia y Tecnología para el Desarrollo" (CYTED). The second author acknowledges support from the Chilean Council of Science and Technology (CONICYT) through the Fondecyt Regular program (project number 1160286).

\section{References}

1. Beliakov G, Pradera A, Calvo T. Aggregation Functions: A Guide for Practitioners. Vol 221. Springer Berlin Heidelberg; 2007.

2. Torra V, Narukawa Y. Modeling Decisions: Information Fusion and Aggregation Operators. Springer Science \& Business Media; 2007.

3. Yager RR, Kacprzyk J, Beliakov G, eds. Recent Developments in the Ordered Weighted Averaging Operators: Theory and Practice. Vol 265. Springer-Verlag Berlin Heidelberg; 2011.

4. Beliakov G, Bustince H, Calvo T. A Practical Guide to Averaging Functions. Vol 329. Springer International Publishing; 2016.

5. Yager RR. On ordered weighted averaging aggregation operators in multicriteria decisionmaking. IEEE Trans Syst Man Cybern B. 1988;18(1):183-190.

6. Yager RR, Kacprzyk J, eds. The Ordered Weighted Averaging Operators: Theory and Applications. Springer US; 1997.

7. Emrouznejad A, Marra M. Ordered weighted averaging operators 1988-2014: A citation-based literature survey. Int J Intell Syst. 2014;29(11):994-1014. 
8. Gil-Aluja J. Elements for a Theory of Decision in Uncertainty. Vol 32. Springer US; 1999.

9. Kacprzyk J. Multistage Fuzzy Control: A Model-Based Approach to Fuzzy Control and Decision Making. 1st ed. Wiley; 1997.

10. Zwick R, Carlstein E, Budescu D V. Measures of similarity among fuzzy concepts: A comparative analysis. Int J Approx Reason. 1987;1(2):221-242.

11. Xu ZS, Chen J. An overview of distance and similarity measures of intuistonistic fuzzy sets. Int J Uncert Fuzz Knowledge-Based Syst. 2008;16(4):529-555.

12. Hamming RW. Error detecting and error correcting codes. Bell Syst Tech J. 1950;29(2):147-160.

13. $\mathrm{Xu} \mathrm{ZS,} \mathrm{Chen} \mathrm{J.} \mathrm{Ordered} \mathrm{weighted} \mathrm{distance} \mathrm{measure.} \mathrm{J} \mathrm{Syst} \mathrm{Sci} \mathrm{Syst} \mathrm{Eng.}$ 2008;17(4):432-445.

14. Merigó JM, Gil-Lafuente AM. New decision-making techniques and their application in the selection of financial products. Inf Sci. 2010;180(11):2085-2094.

15. Merigó JM, Casanovas M. Decision making with distance measures and linguistic aggregation operators. Int J Fuzzy Syst. 2010;12(3):190-198.

16. Merigó JM, Casanovas M. Decision-making with distance measures and induced aggregation operators. Comput Ind Eng. 2011;60(1):66-76.

17. Zeng SZ, Su WH. Intuitionistic fuzzy ordered weighted distance operator. KnowledgeBased Syst. 2011;24(8):1224-1232.

18. Xu ZS. Fuzzy ordered distance measures. Fuzzy Optim Decis Mak. 2012;11(1):73-97.

19. Zhou LG, Chen HY, Liu JP. Continuous ordered weighted distance measure and its application to multiple attribute group decision making. Group Decis Negot. 2012;22(4):739-758.

20. Merigó JM, Xu YJ, Zeng SZ. Group decision making with distance measures and 
probabilistic information. Knowledge-Based Syst. 2013;40:81-87.

21. Zeng SZ, Wei L, Merigó JM. Extended induced ordered weighted averaging distance operators and their application to group decision-making. Int J Inf Technol Decis Mak. 2013;12(4):789-811.

22. Merigó JM, Casanovas M, Zeng SZ. Distance measures with heavy aggregation operators. Appl Math Model. 2014;38(13):3142-3153.

23. Zhou LG, Wu JX, Chen HY. Linguistic continuous ordered weighted distance measure and its application to multiple attributes group decision making. Appl Soft Comput. 2014;25:266-276.

24. Xian SD, Sun WJ, Xu SH, Gao YY. Fuzzy linguistic induced OWA Minkowski distance operator and its application in group decision making. Pattern Anal Appl. 2016;19(2):325-335.

25. Zhou LG, Chen HY. Generalized ordered weighted logarithm aggregation operators and their applications to group decision making. Int J Intell Syst. 2010;25(7):683-707.

26. Zhou LG, Chen HY, Liu JP. Generalized logarithmic proportional averaging operators and their applications to group decision making. Knowledge-Based Syst. 2012;36:268279.

27. Zhou LG, Chen HY, Liu JP. Generalized weighted exponential proportional aggregation operators and their applications to group decision making. Appl Math Model. 2012;36(9):4365-4384.

28. Zhou LG, Tao ZF, Chen HY, Liu JP. Generalized ordered weighted logarithmic harmonic averaging operators and their applications to group decision making. Soft Comput. 2015;19(3):715-730.

29. Shannon CE. A mathematical theory of communication. Bell Syst Tech J. 1948;27(July 1928):379-423. 
30. Yager RR. Families of OWA operators. Fuzzy Sets Syst. 1993;59(2):125-148.

31. Yager RR. Centered OWA operators. Soft Comput. 2007;11(7):631-639.

32. Blanco-Mesa F, Merigo JM, Kacprzyk J. Bonferroni means with distance measures and the adequacy coefficient in entrepreneurial group theory. Knowledge-Based Syst. 2016;111:217-227.

33. Zeng S, Su W, Chen J. Fuzzy decision making with induced heavy aggregation operators and distance measures. J Intell Fuzzy Syst. 2014;26(1):127-135.

34. Merigó JM, Yager RR. Generalized Moving Averages, Distance Measures and OWA Operators. Int J Uncert Fuzz Knowledge-Based Syst. 2013;21(4):533-559.

35. Calvo T, Kolesárová A, Komorníková M, Mesiar R. Aggregation operators: properties, classes and construction methods. Aggreg Oper New Trends Appl. 2002;97(1):3-104.

36. Blanco-Mesa F, Merigó JM, Gil-Lafuente AM. Fuzzy decision making: A bibliometric-based review. J Intell Fuzzy Syst. 2017;32(3):2033-2050.

37. Yu D, Li DF, Merigó JM, Fang L. Mapping development of linguistic decision making studies. J Intell Fuzzy Syst. 2016;30(5):2727-2736.

38. Adams R, Bessant J, Phelps R. Innovation management measurement: a review. Int J Manag Rev. 2006;8(1):21-47.

39. Chiesa V, Coughlan P, Voss CA. Development of a technical innovation audit. J Prod Innov Manag. 1996;13(2):105-136.

40. Damanpour F. Organizational innovation: a meta-analysis of effects of determinants and moderators. Acad Manag J. 1991;34(3):555-590.

41. Bessant J. High-Involvement Innovation: Building and Sustaining Competitive Advantage through Continuous Change. Wiley; 2003.

42. Von Hippel E. Lead users: an important source of novel product concepts. Manage Sci. 1986;32(7):791-805. 
43. Merigó JM, Gil-Lafuente AM, Yager RR. An overview of fuzzy research with bibliometric indicators. Appl Soft Comput. 2015;27:420-433. 
Table I. Results for the alternative formulations of the OWLAD operator

\begin{tabular}{cccc}
\hline OWLAD & OWLAD & OWLAD & OWLAD \\
\hline 9.4162 & 1.7978 & 3.5944 & 1.2468 \\
\hline
\end{tabular}

Table II. OWLAD operator general characterization of the aggregation

\begin{tabular}{lcccc}
\hline Measure & CDA & CDA $^{*}$ & CAA & CAA* \\
\hline Result & 0.4583 & 0.5417 & 0.7287 & 0.2713 \\
\hline
\end{tabular}

Table III. Families of GWLAD operators

\begin{tabular}{clc}
\hline$\lambda$ & Families & Acronym \\
\hline$\lambda \rightarrow 0$ & Weighted logarithmic geometric averaging distance operator & WLGAD \\
$\lambda=-1$ & Weighted logarithmic harmonic averaging distance operator & WLHAD \\
$\lambda=1$ & Weighted logarithmic aggregation distance operator & WLAD \\
$\lambda=2$ & Weighted logarithmic quadratic aggregation distance operator & WLQAD \\
$\lambda=3$ & Weighted logarithmic cubic aggregation distance operator & WLCAD \\
$\lambda \rightarrow \infty$ & Largest of the $\left|x_{i}-y_{i}\right|$ & Max \\
$\lambda \rightarrow-\infty$ & Lowest of the $\left|x_{i}-y_{i}\right|$ & Min \\
\hline
\end{tabular}

Table IIV. Families of the GWLAD operator

\begin{tabular}{cccccccc}
\hline$\lambda$ & $\rightarrow 0$ & -1 & 1 & 2 & 3 & $\infty$ & $-\infty$ \\
\hline Aggregation & 6.1353 & 5.2601 & 7.1682 & 8.2130 & 9.1541 & $\rightarrow 17$ & $\rightarrow 3$ \\
\hline
\end{tabular}


Table V. Results for the alternative formulations of the GOWLAD operator

\begin{tabular}{cccc}
\hline GOWLAD & GOWLAD & GOWLAD & GOWLAD \\
\hline 10.2820 & 1.9220 & 3.9026 & 1.2680 \\
\hline
\end{tabular}


Table VI. Families of GOWLAD operators

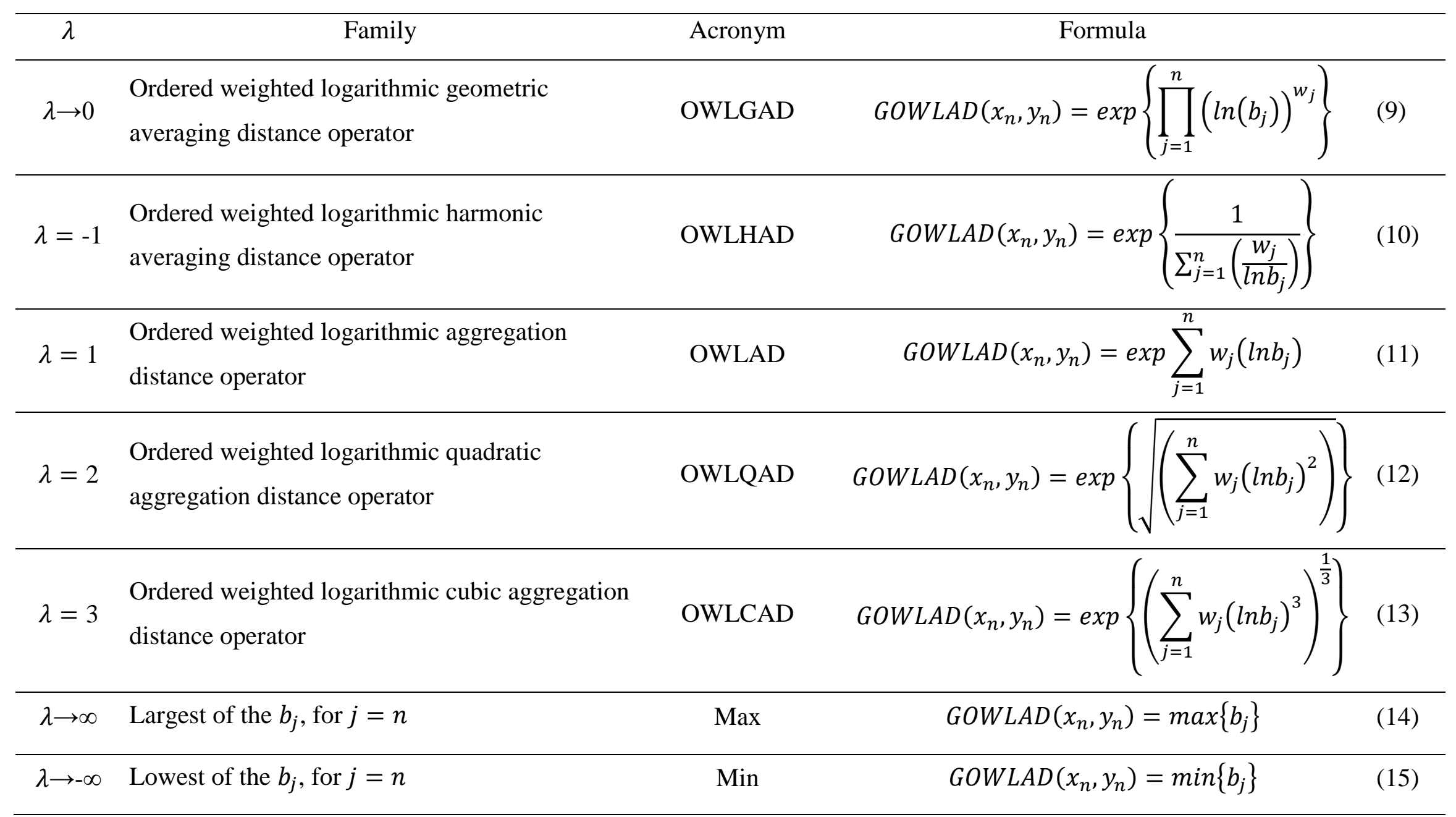

Note that for all cases, $b_{j}$ is the $\left|x_{i}-y_{i}\right|$ value of GOWLAD $\left\langle x_{i}, y_{i}\right\rangle$, in decreasing order of values of $\left|x_{i}-y_{i}\right|$. 
Table VII. Ideal project

\begin{tabular}{lllllll}
\hline & $C_{1}$ & $C_{2}$ & $\ldots$ & $C_{i}$ & $\ldots$ & $C_{n}$ \\
\hline$P$ & $y_{1}$ & $y_{2}$ & $\ldots$ & $y_{i}$ & $\ldots$ & $y_{n}$ \\
\hline
\end{tabular}

Table VIII. Characteristics of the project: valuations from expert 1

\begin{tabular}{lcccccc}
\hline & $C_{1}$ & $C_{2}$ & $C_{3}$ & $C_{4}$ & $C_{5}$ & $C_{6}$ \\
\hline$A_{1}$ & 88 & 56 & 59 & 95 & 90 & 64 \\
$A_{2}$ & 68 & 88 & 69 & 96 & 97 & 96 \\
$A_{3}$ & 95 & 62 & 85 & 99 & 82 & 79 \\
$A_{4}$ & 79 & 62 & 100 & 72 & 67 & 79 \\
$A_{5}$ & 86 & 82 & 100 & 96 & 72 & 58 \\
$A_{6}$ & 60 & 93 & 53 & 59 & 87 & 73 \\
\hline
\end{tabular}

Table IVIII. Characteristics of the project: valuations from expert 2

\begin{tabular}{ccccccc}
\hline & $C_{1}$ & $C_{2}$ & $C_{3}$ & $C_{4}$ & $C_{5}$ & $C_{6}$ \\
\hline$A_{1}$ & 79 & 88 & 76 & 83 & 61 & 85 \\
$A_{2}$ & 63 & 61 & 86 & 68 & 76 & 74 \\
$A_{3}$ & 77 & 86 & 69 & 86 & 71 & 88 \\
$A_{4}$ & 74 & 76 & 66 & 89 & 65 & 62 \\
$A_{5}$ & 61 & 65 & 65 & 84 & 78 & 80 \\
$A_{6}$ & 86 & 73 & 61 & 81 & 85 & 68 \\
\hline
\end{tabular}


Table IX. Characteristics of the project: valuations from expert 3

\begin{tabular}{lcccccc}
\hline & $C_{1}$ & $C_{2}$ & $C_{3}$ & $C_{4}$ & $C_{5}$ & $C_{6}$ \\
\hline$A_{1}$ & 75 & 54 & 75 & 59 & 39 & 35 \\
$A_{2}$ & 33 & 35 & 50 & 92 & 96 & 56 \\
$A_{3}$ & 93 & 63 & 64 & 71 & 38 & 48 \\
$A_{4}$ & 48 & 42 & 70 & 70 & 55 & 77 \\
$A_{5}$ & 61 & 74 & 94 & 61 & 49 & 88 \\
$A_{6}$ & 77 & 90 & 86 & 78 & 35 & 39 \\
\hline
\end{tabular}

Table X. Ideal investment

\begin{tabular}{ccccccc}
\hline & $C_{1}$ & $C_{2}$ & $C_{3}$ & $C_{4}$ & $C_{5}$ & $C_{6}$ \\
\hline$E_{1}$ & 70 & 80 & 100 & 100 & 60 & 80 \\
$E_{2}$ & 90 & 80 & 100 & 90 & 70 & 90 \\
$E_{3}$ & 80 & 90 & 100 & 70 & 50 & 80 \\
\hline
\end{tabular}

Table XI. Collective results in the form of individual distances

\begin{tabular}{ccccccc}
\hline & $C_{1}$ & $C_{2}$ & $C_{3}$ & $C_{4}$ & $C_{5}$ & $C_{6}$ \\
\hline$A_{1}$ & 5 & 19 & 32.75 & 7 & 10 & 20.5 \\
$A_{2}$ & 19.5 & 14.5 & 31.5 & 2 & 31.5 & 2 \\
$A_{3}$ & 12.5 & 14.25 & 24.25 & 1.25 & 8.25 & 9 \\
$A_{4}$ & 7.5 & 22 & 16 & 14.25 & 3.5 & 8.25 \\
$A_{5}$ & 4 & 6.75 & 10.25 & 5.75 & 7.75 & 11.5 \\
$A_{6}$ & 6.75 & 4.75 & 36.75 & 20.75 & 13.5 & 19.25 \\
\hline
\end{tabular}


Table XII. Aggregated results 1

\begin{tabular}{ccccccc}
\hline & Max & Min & NLHD & WLHD & Step (k=3) & WLAD \\
\hline$A_{1}$ & 32.7500 & 5.0000 & 2.5519 & 2.7613 & 3.4889 & 15.8196 \\
$A_{2}$ & 31.5000 & 2.0000 & 2.3218 & 2.4974 & 3.4500 & 12.1508 \\
$A_{3}$ & 24.2500 & 1.2500 & 2.1502 & 2.3586 & 3.1884 & 10.5759 \\
$A_{4}$ & 22.0000 & 3.5000 & 2.3164 & 2.2806 & 2.7726 & 9.7830 \\
$A_{5}$ & 11.5000 & 4.0000 & 1.9771 & 2.1007 & 2.3273 & 8.1718 \\
$A_{6}$ & 36.7500 & 4.7500 & 2.6108 & 2.8433 & 3.6041 & 17.1724 \\
\hline
\end{tabular}

Table XIII. Aggregated results 2

\begin{tabular}{ccccccc}
\hline & GOWLAD -1 & GOWLAD 1 & GOWLAD 2 & GOWLAD 3 & median & olympic \\
\hline$A_{1}$ & 1.0594 & 11.8888 & 3.1291 & 2.1189 & 13.7840 & 12.8499 \\
$A_{2}$ & 1.0407 & 8.3796 & 2.9116 & 2.3097 & 16.8152 & 11.5528 \\
$A_{3}$ & 1.0270 & 7.6037 & 2.6840 & 1.9969 & 10.6066 & 10.7240 \\
$A_{4}$ & 1.0532 & 9.4683 & 2.8235 & 1.9785 & 10.8426 & 10.8984 \\
$A_{5}$ & 1.0471 & 6.7485 & 2.3457 & 1.8549 & 7.2327 & 7.4516 \\
$A_{6}$ & 1.0611 & 12.2402 & 3.1545 & 2.2880 & 16.1206 & 13.8125 \\
\hline
\end{tabular}


Table XIV. Ranking of the performances of the concepts to be developed

\begin{tabular}{lcll}
\hline & \multicolumn{2}{c}{ Ranking } & \multicolumn{2}{c}{ Ranking } \\
\hline Max & $\left.\left.\left.\left.\left.A_{5}\right\} A_{4}\right\} A_{3}\right\} A_{2}\right\} A_{1}\right\} A_{6}$ & GOWLAD $(\lambda=-1)$ & $\left.\left.\left.\left.\left.A_{3}\right\} A_{2}\right\} A_{5}\right\} A_{4}\right\} A_{1}\right\} A_{6}$ \\
Min & $\left.\left.\left.\left.\left.A_{1}\right\} A_{6}\right\} A_{5}\right\} A_{4}\right\} A_{2}\right\} A_{3}$ & GOWLAD $(\lambda=1)$ & $\left.\left.\left.\left.\left.A_{5}\right\} A_{3}\right\} A_{2}\right\} A_{4}\right\} A_{1}\right\} A_{6}$ \\
NLHD & $\left.\left.\left.\left.\left.A_{5}\right\} A_{3}\right\} A_{4}\right\} A_{2}\right\} A_{1}\right\} A_{6}$ & GOWLAD $(\lambda=2)$ & $\left.\left.\left.\left.\left.A_{5}\right\} A_{3}\right\} A_{4}\right\} A_{2}\right\} A_{1}\right\} A_{6}$ \\
WLHD & $\left.\left.\left.\left.\left.A_{5}\right\} A_{4}\right\} A_{3}\right\} A_{2}\right\} A_{1}\right\} A_{6}$ & GOWLAD $(\lambda=3)$ & $\left.\left.\left.\left.\left.A_{5}\right\} A_{4}\right\} A_{3}\right\} A_{1}\right\} A_{6}\right\} A_{2}$ \\
Step (k=3) & $\left.\left.\left.\left.\left.A_{5}\right\} A_{4}\right\} A_{3}\right\} A_{2}\right\} A_{1}\right\} A_{6}$ & Median & $\left.\left.\left.\left.\left.A_{5}\right\} A_{3}\right\} A_{4}\right\} A_{1}\right\} A_{6}\right\} A_{2}$ \\
WLAD & $\left.\left.\left.\left.\left.A_{5}\right\} A_{4}\right\} A_{3}\right\} A_{2}\right\} A_{1}\right\} A_{6}$ & Olympic & $\left.\left.\left.\left.\left.A_{5}\right\} A_{3}\right\} A_{4}\right\} A_{2}\right\} A_{1}\right\} A_{6}$ \\
\hline
\end{tabular}

